\title{
Proportion-based surveillance of antimicrobial resistance remains an essential public health tool for disease control
}

\author{
John W. Tapsall, Gunnar S. Simonsen, Elizabeth A. Talbot and Benedetta Allegranzi
}

A recent opinion ${ }^{1}$ argues that the rate of isolation of antibiotic-resistant organisms, instead of an estimate of their proportion, provides a better measure of the 'burden of resistance' for public health purposes. The authors also suggest that information derived from proportion-based analyses using existing hospital-based antimicrobial resistance (AMR) surveillance systems might lead to biased estimates of AMR that potentially 'mislead public health professionals' and lack comparability.

We too are aware that existing surveillance systems are imperfect, but argue that not all public health needs will be met by determination of rate-based AMR estimates as proposed by Schwaber et al. ${ }^{1}$

Between hospitals, and even within the same hospital, differences in patient mix, available services, clinical and sampling practices and laboratory methods for isolation of organisms and susceptibility testing skew AMR estimates. In resource-poor settings, the capacity for AMR surveillance is limited, and data gathering is biased towards resistant isolates. Many AMR surveillance programmes lack valid quality-assurance components. The compounding effects of these deficiencies preclude useful regional, national or international comparisons of AMR data, whether proportion-based or rate-based. The applications of AMR data analyses are therefore in the main limited to a single institution. Pooling large amounts of data from multiple sources does not remedy these defects and at best provides indicative trends that point to a need for more in-depth studies. Wider public health needs require proportion-based or rate-based surveys that are longitudinal instead of sporadic, use constant and continually validated methods, include data on community-acquired pathogens and meet requirements for sample size and representativeness ${ }^{2}$.

Schwaber et al. ${ }^{1}$ also allude to a 'clinician's perspective', whereby proportion-based AMR analyses are used to change treatments (for example, for Staphylococcus aureus and penicillins, they suggest a threshold for change of $50 \%$ ). We contend that properly performed proportion-based AMR estimates expand and help refine this additional public health principle by providing information relevant to both patient care and population disease control. For diseases such as malaria, tuberculosis, gonorrhoea and shigellosis, the determination of the proportion of antibiotic-resistant strains and the probability of success of standard treatments is an integral part of the disease control process and an important public health tool. The highly successful DOTS and DOTS PLUS programmes for tuberculosis control rely on associated and specific AMR surveillance. A recent publication ${ }^{3}$ linked AMR surveillance to these wider issues of disease control through the use of treatments that interrupt pathogen transmission and eliminate infection reservoirs. Effective treatment of gonorrhoea, determined by AMR surveillance and defined as that single-dose therapy which provides a $95 \%$ cure rate, decreases the incidence of gonorrhoea and its complications such as pelvic inflammatory disease, early trimester abortion and ophthalmia neonatorum ${ }^{4}$. Effective treatment also reduces HIV transmission by eliminating the amplifying effects of gonorrhoea ${ }^{5}$.

Therefore, we state that the focus of Schwaber et al. could be enlarged to include those specific public health needs that current AMR surveillance systems fail to meet, and that proportion-based AMR surveillance remains one essential component of an integrated programmatic approach to disease control.

John W. Tapsall is at the WHO Collaborating Centre for STD and HIV, Prince of Wales Hospital, Randwick, Sydney, Australia 2031.

Gunnar S. Simonsen is at the Norwegian Surveillance System for Antimicrobial Drug Resistance, University Hospital of North Norway, N-9038 Tromsø, and the Norwegian Institute of Public Health, Oslo, Norway.

Elizabeth A. Talbot is at New Hampshire Department of Health and Human Services, and Dartmouth College, New Hampshire, USA.

Benedetta Allegranzi is at the Department of Infectious Diseases, University of Verona, 37126 Verona, Italy. 
1. Schwaber, M. J., De-Medina, T. \& Carmeli, Y. Epidemiological interpretation of antibiotic resistance studies - what are we missing? Nature Rev. Microbiol. 2, 979-983 (2004).

2. Anonymous. Surveillance Standards for Antimicrobial Resistance (World Health Organization, Geneva, 2001).

3. Simonsen, G. S., Tapsall, J. W., Allegranzi, B., Talbot, E. A. \& Lazzari, S. The ARCS approach - a public health tool for antimicrobial resistance containment and surveillance. Bull. World Health Organ. 82, 928-934 (2004).

4. Tapsall, J. W. Antimicrobial Resistance in Neisseria gonorrhoeae (World Health Organization, Geneva, 2001).

5. Cohen, M. S. Sexually transmitted diseases enhance HIV transmission: no longer a hypothesis. Lancet 351, 5-7 (1998).

Competing interests statement

The authors declare no competing financial interests. 


\section{Response}

We appreciate the comments of Tapsall and colleagues regarding proportion-based surveillance of antimicrobial resistance (AMR). We agree with the authors that ratebased estimates of AMR do not meet all public health needs, and that proportionbased estimates continue to have a role in reporting AMR. The examples brought forth by the authors show the usefulness of proportions in situations in which disease treatment and control of pathogen spread are inter-related.

However, even in the examples brought, the value of proportions is primarily in guiding appropriate therapy (referred to in our article as the 'clinician's perspective'). Valid assessments of the success of intervention in eradicating resistant pathogens (the 'public health perspective') necessitate rate-based surveillance data.

Our division of perspectives into those of the clinician and the public health practitioner was done for didactic purposes, to demonstrate pitfalls inherent in reliance on proportion-based data alone. As we stated in our introduction, these perspectives can overlap, as in the examples brought by the authors in which the clinician also plays a vital public health role.

We agree that comparisons among different patient populations may be difficult. Still, such comparisons may be necessary, and in our paper we offered suggestions to overcome some of these difficulties, including the use of case-mix indicators and adjusted analyses.

One final point: our example of a physician treating suspected Staphylococcus aureus infection in the intensive-care unit was not intended to suggest a threshold proportion of resistance at which use of vancomycin would be indicated. Such thresholds should be determined based on the specific clinical syndrome involved as well as the usefulness and toxicity of the antibiotics in question.

\section{Mitchell J. Schwaber, Tali De-Medina and Yehuda Carmeli}

Mitchell J. Schwaber and Tali De-Medina are at the Division of Epidemiology, Tel Aviv Sourasky Medical Center, Tel Aviv 64239, Israel.

Yehuda Carmeli is at the Division of Epidemiology, Tel Aviv Sourasky Medical Center, Tel Aviv 64239, Israel, and the Division of Infectious Diseases, Beth Israel Deaconess Medical Center, Harvard Medical School, Boston, Massachusetts, USA. 